\title{
LA AUTOETNOGRAFÍA INTERPRETATIVA PARA HUMANIZAR LA INVESTIGACIÓN SOCIAL EN CONTEXTOS TRANSFRONTERIZOS LATINOAMERICANOS ${ }^{1}$
}

\author{
INTERPRETATIVE AUTOETHNOGRAPHY AS A QUALITATIVE METHODOLOGY TO HUMANIZE \\ SOCIAL RESEARCH IN LATIN AMERICAN TRANSBOUNDARY CONTEXTS
}

AUTO ETNOGRAFIA INTERPRETATIVA COMO METODOLOGIA PARA HUMANIZAR

A INVESTIGAÇÃO SOCIAL EM CONTEXTOS FRONTERIZOS LATINOAMERICANOS

\author{
Pamela Zapata Sepúlveda \\ Universidad de Tarapacá. Arica, Chile. \\ pzapatas@uta.cl \\ ORCID 0000-0003-3633-5673
}

DOI: https://doi.org/10.22235/ech.v6iEspecial.1451 Recibido: $15 / 06 / 2017$

Aceptado: 17/08/2017

\section{RESUMEN}

Este manuscrito tiene como objetivo conectar el momento actual de la Investigación Cualitativa contemporánea con la audiencia de investigación cualitativa latinoamericana desde una aproximación interdisciplinaria. Para ello se expondrán los principales enunciados que sitúan la investigación cualitativa desde la tradición de la autoetnografía interpretativa, entendida como una forma de investigación crítica que busca, entre otros fines, sensibilizar, evocar y transformar realidades a partir de la escritura experimental como forma de investigar. Esta metodología, ampliamente empleada en proyectos de investigación en países angloparlantes para abordar injusticias y problemas que afectan las vidas de personas sin voz, permite relevar conocimientos desde lo auto, lo etno, a lo social. En el presente manuscrito se realiza una reseña bibliográfica acerca del método y un ejemplo tomado a partir de la experiencia de trabajo de campo en el proyecto Fondecyt regular № 1160869 "Relaciones e interacciones sociales de niños hijos de inmigrantes y niños chilenos en las escuelas de Arica". Se discute las aplicaciones y los aportes de esta metodología para la investigación social desde la voz de una mujer latinoamericana que desarrolla su línea de investigación desde una región fronteriza, y cómo estas metodologías pueden atender a los cuidados de los participantes de los estudios.

Palabras clave: Autoetnografía Interpretativa, Texto Experimental, Storytelling, Investigación Interdisciplinaria, Migraciones e Infancia, Vida Cotidiana.

\section{ABSTRACT}

This paper aims to connect the present moment of contemporary Qualitative Inquiry with the Latin American qualitative inquiry audience from an interdisciplinary approach. In order to do so, the main statements that place the QI in present times will be presented, specifically the tradition of interpretative autoethnography understood as a form of critical research that seeks to sensitize, to evoke and to transform realities through experimental writing as a way of investigating. This methodology, widely used in research projects in English speaking countries to address injustices and problems that affect the lives of voiceless people, allows to relay knowledge from the self, the ethno, to the social. In this paper, a bibliographical review about the method is conducted and addresses an example taken from field work experience in the project Fondecyt regular № 1160869 "Relationships and social interactions of children of immigrants and Chilean children in the schools of Arica". The applications and contributions of this methodology for social research are discussed through the voice of a Latin American woman who develops her research line from a border region, and how these methodologies can address the caretaking of the participants of the study.

Keywords: Interpretive Autoethnography, Experimental Text, Storytelling, Interdisciplinary Research, Migration and Childhood, Everyday Life.

\section{RESUMO}

Este trabalho tem como objetivo conectar o momento atual da Investigação Qualitativa, com o público da investigação qualitativa latino-americana, desde uma perspectiva interdisciplinar. Para isso serão expostos os principais e atuais conceitos que situam a IQ, especificamente a tradição da autoetnografia interpretativa, entendida como uma forma de investigação crítica que busca entre outros fins, sensibilizar, evocar e transformar realidades a partir da escrita experimental como forma de in-

1 Este manuscrito forma parte del proyecto de investigación FONDECYT - Regular (2016-2018), № 1160869: Social relations and interactions among the children of immigrants and the children of Chileans in schools in Arica: Construction of a habitus in everyday school life. 
vestigar. Esta metodologia amplamente empregada em projetos de investigações em países de língua inglesa, para abordar injustiças e problemas que afetam as vidas das pessoas sem voz, permite revelar conhecimentos desde o auto, o etno até o social. No presente trabalho, realiza-se uma resenha bibliográfica acerca desse método e um exemplo tomado a partir da experiência do trabalho de campo no projeto Fondecyt regular № 1160869 "Relações e interações sociais de crianças filhas de imigrantes e crianças chilenas nas escolas de Arica." Discutiremos as aplicações e os aportes desta metodologia para a investigação social, desde a voz de uma mulher latino-americana que desenvolve sua linha de investigação em uma região fronteiriça, e como estas metodologias podem atender aos cuidados dos que participam desses estudos.

Palavras-chave: Autoetnografia Interpretativa, Texto Experimental, Storytelling, Investigação Interdisciplinar, Migrações e Infância, Vida Cotidiana.

\section{INTRODUCCIÓN}

Hacer investigación cualitativa en universidades latinoamericanas no es una tarea sencilla. Aunque han pasado décadas de las tan conocidas disputas entre los investigadores cualitativos y cuantitativos en los años 80 , en lo cotidiano estas disputas siguen estando presentes en el ámbito de la investigación en ciencias sociales y en salud en el sistema actual de educación superior en Latinoamérica. En ese ámbito el número vale más que el contenido y la relevancia de los problema que se estudian, en un sistema mundial que valora una carrera académica basada en la cantidad de papers, los rankings e indexaciones, más que en el valor que un proyecto de investigación pueda tener para una comunidad $(1,5,6)$.

En el contexto latinoamericano actual de la investigación en áreas como las ciencias sociales y la salud, nos encontramos con paradigmas y formas de entender la investigación que se imponen y contraponen, con criterios sobre lo científico basado en lógicas positivistas que difícilmente pueden dialogar con la investigación cualitativa que releva el conocimiento local a partir de las voces de las personas que son investigadas. Al mismo tiempo, nuestras voces en investigación deben quedar silenciadas, existiendo la tendencia predominante a hacer investigación exclusivamente a partir de los datos que nos entregan los participantes (entrevistas, focus groups, etc.).

Así, los investigadores cualitativos en Latinoamérica subsistimos con menores posibilidades de publicación en revistas latinoamericanas, y menores aún en revistas ISI de países que predominan en cuanto a producción científica, desde donde se critica incluso el desarrollo de producciones científicas en Latinoamérica a partir de réplicas de estudios realizados en estos países (ejemplo, los Estados Unidos). De ahí se releva la importancia de crear nuestras propias lógicas metodológicas y criterios de rigor no adaptados de los países predominantes en ciencia. Esto, que se plantea como una crítica a la ciencia en Latinoamérica, requiere de la consideración del contexto en cuanto a los ajustes socioeconómicos e institucionales necesa- rios al hacer una investigación (7). Así, y como plantea Spinak, el contexto social del proceso científico moldea la selección de temas de investigación y publicación. Debido a esto, la investigación en ciencias sociales y salud debería subrayar nuestras prioridades y objetivos que se expresan primero en nuestros propios idiomas, en donde los análisis fuera de contexto, aplicados a los esfuerzos de científicos latinoamericanos, podrían tender inevitablemente a la alienación. Esto "si en nuestros esfuerzos científicos frecuentemente imitamos modas y buscamos un reconocimiento que tenga sentido sólo en el contexto cultural y social de los países centrales" (7).

Entonces, "si, como algunos creen, las actividades científicas sólo pueden desarrollarse dentro de los paradigmas elegidos por los países ricos, la consecuencia es que los países latinoamericanos quedan relegados al rango de colonias científicas.", ". . . confundiendo la comunidad científica internacional con la del mundo angloamericano, convirtiéndose esta última en la única fuente de normas y criterios para conceder o negar la validez. . . " a nuestros esfuerzos científicos, viéndonos forzados a tener que internacionalizar nuestros trabajos sobre realidades locales para lograr publicarlos en el idioma inglés, el lenguaje de la ciencia, a través de plataformas académicas internacionales, logrando el éxito esperado en audiencias muchas veces lejanas a las realidades que estudiamos (Conferencia de Etnografías Contemporáneas a través de las Disciplinas [CEAD]) (7). En esta carrera tenemos que lidiar con las políticas de ciencia y tecnología gubernamentales que, para el caso de Chile, no financia ensayos de investigación sino reportes de resultados de investigación, y en los que la aprobación de proyectos de investigación cualitativa se corresponda más bien a los métodos mixtos de investigación con el uso de programas informáticos, frecuencias, análisis de conglomerados, etc.

De acuerdo a lo anterior, la tendencia en investigación cualitativa latinoamericana responde a estas exigencias y responden al momento 8 de la IC (1). En los Estados Unidos y países angloparlantes como Inglaterra, Australia, y Nueva Zelanda, se evidencian nuevas (para nosotros en Latinoamérica) líneas de investigación cualitativa ya instauradas desde hace aproximadamente 20 años (8 - 12). El desfase de momentos al que hago mención queda en evidencia cada año en el congreso mundial de investigación cualitativa que se celebra en la University of Illinois at Urbana-Champaign liderado por el Dr. Norman Denzin, y en la conferencia de Etnografías contemporáneas a través de las disciplinas que se traslada por el sur del mundo cada dos años y se celebrará en 2018 en Santiago de Chile.

Un reflejo de ello podemos encontrar cuando asistimos a una conferencia de investigación cualitativa y podemos diferenciar los distintos enfoques, metodologías y paradigmas de los investigadores latinoamericanos y los de países anglo. Desde mi trayectoria investigativa realizando estudios en Chile desde 1999 en Arica , 2002 - 2006 en España, y desde 2009 en los Estados Unidos, y asistiendo a conferencias nacionales e internacionales desde el año 2002, he podido constatar que lo que entendemos por IC en Latinoamérica corresponde a un momento anterior a cómo se está haciendo la IC en países de habla inglesa como en los Estados Unidos (13-15). En este sentido, Denzin y 
Lincoln sostienen que en estos tiempos "necesitamos mejorar las nuevas formas de conectar a las personas y sus problemas personales con metodologías de justicia social. Necesitamos llegar a un mejor logro en la vinculación de estas intervenciones a aquellos sitios institucionales donde los problemas se convierten en cuestiones públicas y los asuntos públicos transformados en políticas sociales" (1). Específicamente, el llamado para el encuentro de este 2017 es "La Investigación cualitativa en la esfera pública". "La investigación cualitativa crítica está bajo asalto. Estos son tiempos difíciles. La derecha global está en movimiento. Establece la agenda del discurso público sobre el bien social. Al hacerlo, está reduciendo los espacios para el discurso cívico. Un reino de miedo está en el aire. EI XIII Congreso Internacional ofrece a los académicos la oportunidad de resistir este discurso, de experimentar diferentes modos de ser moral, político y ético en la esfera pública, de poner en primer plano, de interrogar e inventar nuevas prácticas interpretativas, de participar en una política de abogacía, pro y con, formar coaliciones, experimentar nuevas formas de resistir las presiones del neoliberalismo. El Congreso será un escenario para promover las causas de la justicia social, al tiempo que aborda las disparidades raciales, étnicas, de género y ambientales en educación, bienestar y salud" (16).

En este recorrer y como se puede observar en el programa de los trabajos que presentan cada año los investigadores cualitativos en Urbana-Champaign, se puede constatar que la tendencia actual en Investigación Cualitativa se basa en las artes, la autoetnografía, psicología crítica postestructuralista, investigación cualitativa crítica, herramientas digitales para la IC, investigación indígena, investigación cualitativa global en salud, entre otros grupos que buscan en común producir transformación social en los entornos de trabajo de sus miembros. En este sentido, se trata de una investigación cualitativa que no se limita al uso de técnicas de recolección de datos sino que su sustento epistemológico se encuentra en teorías filosóficas de base que trabajan en o junto a la tradición continental: "Agamben, Badiou, Deleuze, Derrida, Foucault, Heidegger, Lacan, Malabou, Mouffe, Nancy, Nussbaum, y Žižek" (Fuente página web del International Congress of Qualitative Inquiry - sección último grupo).

Reconociendo que en la academia actual la lucha por la justicia social tiende a ser un trabajo en solitario, en entidades que se basan en la eficiencia cooperativa, esta labor trata de un desafío ante los "gobiernos neoliberales alineados con las corporaciones multinacionales", y entonces el llamado -o el motor- es a formar parte de la comunidad de ICQI y trabajar juntos por la justicia social de nuestras comunidades.

Hacer este tipo de investigación en latinoamérica no es usual. Al buscar en la bibliografía se puede ver que los investigadores latinoamericanos que desarrollan alguna de las modalidades de autoetnografía suelen ser académicos mexicanos que han recibido formación especializada en los Estados Unidos. Ejemplo de ello son los trabajos de Elizabeth Aguirre-Armendariz y Silvia Bénard Calva, o de otros académicos latinoamericanos que trabajan en universidades de los Estados Unidos como los brasileños Marcelo Diversi y Claudio Moreira (17- 19). Por otro lado, existe una amplia gama de textos de escritores latinoamericanos, que sin ser investigadores cualitativos, aportan elementos literarios desde el pensamiento y las realidades latinoamericanas.

Aún más difícil es encontrar a investigadores latinoamericanos que desarrollen autoetnografía interpretativa o estudios de performances. En este sentido, y estudiando el método en el centro de investigación cualitativa del profesor Norman Denzin, padre de la autoetnografía interpretativa, tras desarrollar este tipo de trabajos durante los últimos siete años considero que hacerlo en contextos latinoamericanos es trasgresor, inusual, inédito, y como plantea Pelias, comprende una fractura al discurso académico predominante en investigación; y para el terreno de la educación, se trata de un marco de investigación disruptivo acerca del pensamiento social crítico como camino para "ser, investigar, escribir, asesorar, y enseñar investigación cualitativa en la academia" (20-21).

La escuela de la Autoetnografía interpretativa parte de supuestos tales como:

Charles Wright Mills sostiene que la imaginación sociológica nos habilita para comprender la historia y la biografía, y la relación entre las dos en la sociedad. El desafío, como plantea Denzin, es desarrollar una metodología que nos permita examinar cómo los problemas privados de los individuos están conectados a asuntos públicos y las respuestas públicas a estos problemas. Esa es su tarea y su promesa. Las personas pueden entender su propia experiencia y evaluar su propio destino únicamente localizándose a sí mismas dentro de su período de momento histórico (3).

Para Denzin, el método trata de un trabajo diseñado para proporcionar a los estudiantes y profesores una descripción accesible de un enfoque crítico, existencial, interpretativo, que ha sido practicado en sus trabajos y el de otros:

"La biografía interpretativa, que en las dos últimas décadas ha sido asombrosamente proliferado como métodos (auto)biográficos interpretativos, Richardson llama prácticas creativas analíticas. Estas prácticas, se edifican y mueven a través de las clásicas versiones de historias de vida y estudios de caso biográfico. E incluyen etnografía narrativa, la meta autoetnografía, la autoetnografía, la autoetnografía colaborativa, la autoetnografía desconolizada co-construida, la duoetnografía, la escritura colaborativa, el etnodrama, la etnografía performativa, la sociopoesía, la escritura performativa, la escritura de historias, la ficción etnográfica, el texto polivocal, y mis historias" (3).

“...Estas nuevas formas de historias de vida toman forma en los términos clave que les definen: Narrativa, significados, voz, experiencia, reflexividad, presencia, representación" (3).

Para los investigadores críticos estos términos son considerados como restos de una época de la investigación humanística que acríticamente valorizó el yo y sus experiencias sociales (Jackson y Mazzei; McLure; Pollock; Scott; St. Pierre \& Pillow; otros) (3). En este sentido la investigación cualitativa en latinoamérica 
sigue de alguna manera estas tendencias, a dejar fuera de los proyectos de investigación tanto las biografías como la voz en primera persona, centrándose en los reportes de resultados. En paralelo, mientras así transcurre la investigación cualitativa en países de latinoamérica, las representaciones en línea de las experiencias de vida proliferan y el self virtual con sus historias de vida están presentes en todas partes gracias a las tecnologías de los medios de comunicación sociales (Skype, facebook, twitter, youtube, etc.).

En escenarios latinoamericanos como el de la región fronteriza de Arica y Parinacota en Chile, la investigación en ciencias sociales aborda la voz y la presencia de las personas indígenas en espacios colonizados, que, como plantea Smith, giran en torno a historias orales, mitos y narrativas performativas para dar sentido a sus vidas, a sí mismos, y las historias colectivas. En estos escenarios, las palabras, los rituales, y las performances son importantes para ellos (3).

A pesar de la historia y las culturas que conforman las sociedades que son estudiadas, la voz de los investigadores sigue siendo la del investigador basado en lógicas positivistas que se esfuerza por ser neutral para llegar a sus resultados siguiendo una lógica lineal, omite su color y su cultura, o se ve a sí mismo y se relaciona con su objeto de estudio desde la mirada imperial de una persona blanca occidental y no indígena, que estudia a otro subordinado, aproblemado, carente, deficitario, pobre. Así, las ciencias sociales siguen siendo procesos de colonización a partir de las tendencias mundiales en investigación en el mundo occidental. Así, las ciencias sociales latinoamericanas y la investigación cualitativa parten de más supuestos cuantitativos que cualitativos, importados de los países que dominan la ciencia, y nosotros los investigadores no necesariamente somos conscientes de qué tanto de ello hay en nuestros proyectos de investigación.

De acuerdo a lo anterior, para el caso de la psicología en la región desde la que escribo, no contamos con una asignatura de psicología indígena latinoamericana que aborde rigurosamente el estudio teórico y metodológico de las experiencias de vida particulares de las personas de culturas indígenas que son estudiadas, y considere las distintas realidades históricas y socioculturales en que estas experiencias se desarrollan, entendiendo el lenguaje local, y el desentramado de las características locales desde una visión nativa. En otras palabras, no contamos con teorías e investigación que incluya un cuerpo de conocimientos con conceptos indígenas, metodologías indígenas, pruebas de personalidad indígenas, nuevas líneas de enseñanza de psicología indígena, y una participación activa en las comunidades indígenas que nos permita localizar el campo desde una perspectiva descolonizada (22-23).

Por su parte, Denzin sostiene que "en las tres últimas décadas en los Estados Unidos, ha resurgido un interés en aproximaciones interpretativas para el estudio de la cultura, la biografía, y la vida de grupos humanos" (3). Un punto central a esta visión ha sido el argumento de que las sociedades, las culturas y las expresiones de la experiencia humana pueden ser leídas como textos sociales, como estructuras de representación que requieren de asuntos simbólicos (Clifford y Marcus; Panourgia y Marcus; Van Maanen). Estos textos, orales o escritos, han tomado un estatus problemático en el proyecto interpretativo (Conquergood). Entonces las preguntas han emergido concernientes a cómo los textos son declarados, leídos, e interpretados (Derrida), y en cómo ahora tanto autores, vidas, sociedades, culturas, y la expresión de la experiencia humana son el principal tópico que se debate en textos interpretativos (Geertz). En el método autobiográfico interpretativo - una vida puede ser capturada y representada en un texto -ahora abierto a preguntas. "Una vida es un texto social, una producción ficticia y narrativa".

La propuesta de Denzin refiere un nuevo camino para explorar un nuevo set de términos. Pasar desde la tradicional historia de vida a un proyecto biográfico en un proyecto autoetnográfico interpretativo. En prácticas críticas, performativas, que comienzan con la biografía del escritor y se mueven hacia la cultura, el discurso, la historia y la ideología. "La autoetnografía performativa interpretativa permite al investigador tomar cada vida personal en su inmediata particularidad y en el terreno de su vida en su momento histórico. Los eventos ocurren en estos sitios donde la estructura, la historia y la autobiografía intersectan" (3).

Aunque emplear una nueva metodología creada en los Estados Unidos parece contrario a lo que sostengo en este mismo texto, y mantengo la necesidad de generar conocimientos locales para estudiar nuestras comunidades locales, desde metodologías decolonizadoras, considero que las "armas" metodológicas que incluye el método en cuestión están inicialmente dadas, pero el contenido, la voz propia del investigador y los asuntos que son abordados, obligan al investigador a desarroIlar un arduo, muchas veces solitario y vulnerable proceso para encontrar su propia voz y reescribir las historias y los conocimientos de sus investigaciones convencionales desde una visión no colonizada.

De acuerdo a lo anterior, asistir al encuentro mundial de investigación cualitativa que se realiza cada año en la Universidad de Illinois en Urbana-Champaign permite conectar los mundos en la investigación y validar nuestras voces en un discurso internacional. En esto tenemos mucho por hacer. Generar nuevas formas de escritura experimental, que, por lo demás, se rigen por las normas de nuestro idioma y consecuentemente, nuestras formas de pensar. Incorporar, resignificar y reescribir conocimientos situados desde paradigmas que podamos formular desde los conocimientos locales que relevamos. Ahí está el desafío. No en convertirnos en una copia minoritaria y periférica que subsiste de manera desventajada en relación a investigadores del "primer mundo", sino que, por el contrario, valorar la riqueza local, tomar elementos teóricos y metodológicos de países que han desarrollado trayectorias reconocidas internacionalmente, y desarrollar nuevas formas de conocimiento que incluyan el reconocimiento de nuestras motivaciones, posiciones en el campo y que permitan hacer investigación social crítica que tenga un impacto positivo en la vida de las personas que estudiamos. 
En este sentido, los aportes de la autoetnografía interpretativa para el estudio de problemáticas de frontera en la macro región centro-sur andina desde donde se desarrolla el proyecto Fondecyt № 1160869 nos han permitido incorporar nuestras voces en primera persona y nuestras biografías a través de formas propias de pensar, analizar, problematizar y comunicar, para responder a nuestras preguntas generadas antes y durante las fases iniciales de la investigación y el trabajo de campo, sobre, por ejemplo, quiénes son los sujetos que estudiamos y qué significados tiene en la región ese "otro", "nosotros", "migrante", o "hijo de inmigrante", "extranjero", en relación a un "nosotros"; para comprender el contexto transnacional en el que se desarrollan nuestras vidas. En este sentido, se trata de hacer investigación que tensione los conocimientos sociológicos, psicológicos, históricos y antropológicos a la base del proyecto, con la realidad desde una perspectiva descolonizada de saberes.

No obstante, el riesgo en el uso de estas metodologías está en que estas nuevas formas de investigar se conviertan en una moda que de alguna manera garantiza sintonizar con lo que los editores norteamericanos esperan como producto publicable en revistas indexadas tipo ISI. En este sentido, considero que hacer autoetnografía interpretativa o alguna de sus formas requiere de un largo proceso de aprendizaje que obliga a conectar y situar el propio self con el self de los otros en ese espacio particular social, político, cultural donde se desarrolla la vida de las personas que investigamos, y entre ellos de las personas que investigamos.

En mi experiencia, comencé a instruirme en este tipo de metodologías en el International Center for Qualitative Inquiry del profesor Norman Denzin en la University of illinois at Urbana Champaign, asistiendo de manera regular desde tres a siete meses por año ininterrumpidamente desde el año 2012. El profesor Denzin en la primera estancia de investigación me recomendó dos libros que fueron clave para lo que denomino descolonizar mi cerebro para el estudio del trauma de ex presos políticos chilenos. Estos libros fueron: Life After Leaving: The Remains of Spousal Abuse (Writing Lives: Ethnographic Narratives) y Memory Is Another Country: Women of the Vietnamese Diaspora, escrito por Nathalie Huynh Chau Nguyen (24-25).

De alguna manera, y sin ser muy consciente del proceso que comenzó con mi primera estancia en Urbana-Champaign, este proceso significó en mí una nueva forma de hacer investigación en profundidad, pero también una nueva forma de ver la vida y de relacionarme con las personas. Una forma de ver con otros ojos los paradigmas que empleaba y las formas de hacer investigación que para mí trataban de la investigación cualitativa, pero que ahora no lo eran del todo y más bien se aproximaban a los métodos mixtos de investigación en ciencias sociales y en salud.

En este recorrido, hacer este tipo de metodologías en contextos latinoamericanos, en un principio sobre las dolorosas experiencias traumáticas producto de las experiencias de represión política en Chile entre 1973 y 1990, significó pasar de relacionarme con las personas que estudiaba desde una postura de observadora externa - que analizaba datos con CAQDAS a partir de sus discursos obtenidos a través de las entrevistas semiestructuradas que les realizaba, a situarme en/ entre/con/desde los supervivientes a estas experiencias, incorporando tras un proceso de revelación y darme cuenta de mi biografía como hija/niña/mujer/ estudiante/académica, nacida y criada en dictadura $(15,26)$.

Como investigadores, no siempre somos conscientes de las decisiones que tomamos al hacer investigación y las conexiones de nuestra biografía como base de nuestros intereses en investigación; tampoco de nuestras formas de entender la realidad y de las influencias teóricas y metodológicas que tenemos encorporizadas en nuestro yo. En este proceso personal, la relevancia de la propuesta metodológica de Denzin para el estudio de los proyectos que he desarrollado, originalmente sobre los procesos de traumatización de ex presos políticos a más de 30 años de ocurridos los hechos, el trauma en las vidas de mujeres colombianas que llegan a Chile huyendo de la guerrilla a principios del 2010 por la frontera del extremo norte de Chile (27 - 29), y hoy en el estudio de las interacciones y las relaciones sociales de niños hijos de inmigrantes en la ciudad fronteriza de Arica, radica en que ha permitido crear narrativas de resistencia, historias autoetnográficas que al mismo tiempo que resisten, demandan ser contadas (3). Se trata de historias que parten de epifanías o momentos reveladores de la investigación y que performan realidades desde un lugar de dolor, un self escrito como un yo performativo, un self que resiste, dice, escapa, siente desde la relación con el otro que participa de la investigación.

Estas historias permiten dar cuenta precisa de experiencias que se mueven desde las voces de personas sin voz pero que paradójicamente las cuentan, y hacia grupos - las audiencias - que conectan con las vidas de investigadores e investigados, dando estructura y significados a las vidas que están siendo estudiadas. Se trata de las acciones que se registran en la observación participante, también las omisiones y secretos, miradas que envuelven información relevante sobre lo que no se dice, aunque la "relevancia" abre todo un universo de matices culturales y éticos. En este sentido, como plantea Muñoz, "las palabras no dichas y los acuerdos tácitos son, muy a menudo, la comunicación humana en su forma más compleja y elegante" (30).

Desde una posición descolonizada, quienes deberían tener la última palabra sobre los problemas que ocurren en sus vidas son las mismas personas que están siendo estudiadas. El resultado de estudios de autoetnografía interpretativa, performan las historias de las personas con y para ellos.

Asimismo, hacer investigación social crítica como la autoetnografía interpretativa en contextos académicos latinoamericanos abordando nuestros problemas de violencia cotidiana, racismo, discriminación en escenarios académicos en que las ciencias sociales siguen enseñándose desde y para población no indígena, las experiencias del investigador cualitativo se incorporan como notas de campo y no como resultados de una investigación (31). La posición en el campo de enseñanza/investigación/aprendizaje, tiende a valorar la neutralidad de por qué hacemos lo que hacemos, sin poder situarnos con 
nuestros estudiantes incorporando en los conocimientos que impartimos nuestras biografías; hace que esta opción metodológica trate a lo menos de cierta valentía para implicarse en los proyectos de investigación que desarrollamos, la necesidad de estar presente en cada fase del proceso, desarrollar una capacidad reflexiva crítica para cuestionar nuestros propios paradigmas aprendidos en la academia para investigar, y situarnos con claridad en un campo que muchas veces es minado por los gobiernos, las lógicas neoliberales en investigación, ciencia y tecnología en nuestros contextos académicos latinoamericanos, y un sistema jerárquico que de alguna manera controla las voces principalmente de mujeres en la academia.

Al mismo tiempo, hacer este tipo de investigación sobre estos problemas en Latinoamérica produce un discurso que quiebra el discurso académico convencional con la frescura de la vivencia sensorial que facilita la comunicación con la audiencia, como forma de construir nuevas realidades viéndonos más cerca que lejos de las personas que investigamos. En este sentido, si pensamos en los aportes de la investigación a la sociedad, este tipo de metodologías permite desde momentos específicos e historias individuales conectarnos como seres individuales universales, y desde ahí comprender al otro oprimido que sufre en este caso, niños y niñas que vienen sin haberlo decidido acompañando a sus padres a un país distinto que ahora es también su país.

El texto performativo encarna y hace la teoría y la experiencia visible para otros (32). Es encorporizado, evocativo, siempre inconcluso e ilimitado (33). Para Elbaz, los textos autoetnográficos son escritos en una perspectiva doble en mente para "otro" (padre, hermano, teórico, autor, audiencia, etc.) (3). Estos textos son producciones también ideológicas de clase, género, "raza", en un momento determinado por la historia, la cultura, el acontecer; en este tipo de investigación cualitativa trata de aspectos que cobran relevancia para entender la experiencia humana, donde el conocimiento visceral puede existir en toda su fisicalidad (34).

\section{A continuación un ejemplo.}

Historia 1. Año 1984. Mi historia (27).

Karen (este y todos los nombres son ficticios) es una amiga de la infancia con quien asistíamos diariamente a clases en el centro de la ciudad. Nosotras, junto a Cristina, Dayffa, Whanda, Berta y Marcela, nuestras otras amigas, éramos estudiantes de un colegio católico de mujeres en los 80 . Nuestra infancia transcurría en perfecta tranquilidad. Pienso en aquella época y vienen a mí el sonido de nuestras risas, el timbre agudo de nuestras voces chillonas, las largas sesiones de intercambio de cartas, y de todo lo que se nos ocurría coleccionar. El álbum de Frutillitas fue uno de los que primero llené y llegué a tener tantas láminas que lo llené dos veces! Nuestras colecciones de esquelas, hojas con dibujos, flores, ángeles. También las servilletas que tía Olga, una tía soltera cuñada de la tía María - hermana de mi mamá -, traía de los Estados Unidos cada vez que nos venía a visitar. Las esquelas de color rosado siempre tenían mayor valor y yo era muy buena para negociar y conseguir las esquelas más bonitas. Aún conservo esa colección de esquelas y también las servilletas. En aquella época, definitivamente no recuerdo haber sido una alumna con un desempeño destacado; agradezco a mis padres que aunque hicieron que nunca me faltara nada para estudiar, no tenían las aprensiones, aspiraciones e intereses de los padres de mis compañeras, que en general tenían como imperativo obtener buenas notas. Recuerdo que estas compañeras lloraban si obtenían la nota máxima, 7.0, o una nota mínima para aprobar, 4.0.

En la enseñanza básica yo iba a jugar al colegio. No me iba mal, porque aunque siempre he sido un tanto distraída, una parte de mí atendía en clases y bastaba un poco de esfuerzo para aprobar cada curso. Con mis amigas siempre corríamos por el patio más amplio de mi colegio, rodeado por salas, gradas y altos muros de concreto, todo en perfecta pulcritud. Esto era típico en colegios de religiosas, que suelen asegurar a los padres la máxima protección y bienestar para quienes estudiábamos allí.

En Arica, una ciudad en la que prácticamente nunca llueve y el frío de invierno es muy llevadero, recuerdo aquella etapa de mi vida que se inició a los 8 años y hasta los 17. Eran días tan soleados, en una época en que los problemas actuales como la radiación solar no estaban presentes y nada parecía impedir nuestra sensación de libertad para jugar hasta que sonaba el timbre que anunciaba que teníamos que regresar a la sala de clases. Paralelamente, en aquella época, una parte importante de la población chilena - también en Arica- vivía las peores atrocidades de la violencia y la tortura por motivos políticos en Chile (35). Pero nosotras no éramos conscientes de lo que pasaba afuera, aunque algo de ello nos impactaba desde la estructura social en la que estábamos siendo partícipes en nuestros roles de estudiantes.

Secretos, silencios, miradas de preocupación, omisiones por parte de mis amigas sobre nuestras historias familiares, parecían quedar fuera del colegio. Hoy, conversando con mis amigas de toda la vida, ¿cómo podía una niña de 7 años decir que su padre no era su padre, si era un requisito de ingreso al colegio el ser hija de padres casados? ¿Cómo podía alguna de nosotras en la adolescencia hablar de sexualidad si esto incluso hoy en el año 2017 sigue siendo un tema que ha generado una serie de debates aún sin resolver? En aquella época, reconocer el origen étnico era reconocerse sin derechos, un descrédito, una vergüenza. Entonces, ahora entiendo cómo la ausencia de historias familiares sobre nuestro origen local fue una medida familiar que así como aquellos altos muros que rodeaban el colegio, garantizaban protección a nuestras vidas de niñas en contra de la violencia y la discriminación.

En esta aparente infancia protegida, como he escrito anteriormente, transcurrían nuestras vidas (27). Allí, Karen, que era más risueña que yo y con quien a veces no podíamos parar de reírnos en clases, siempre era descubierta por la profesora de inglés. La profesora, cada vez en menos tiempo desde el inicio de su clase terminaba pidiéndole a Karen en tono molesto que se retirara de la sala. Este era el momento en que la sonrisa de Karen y también la mía, se apagaban. En mi caso, recuerdo que mis manos comenzaban a sudar, con el temor de que 
la profesora me hubiese visto riendo también, y entonces me pidiera que me retirara de la sala, y me enviara a la inspectoría del colegio, en donde tenía que permanecer a veces escuchando un discurso de reclamo, con una dosis de culpas, y asumiendo un rol encasillado y difícil de sacarse del cuerpo, el de mala estudiante. Esto era lo que le pasaba a Karen cada vez que la profesora de inglés pedía que se retirara de la sala. ¿Qué peor situación puede ocurrir para una niña de $8-9$ años que ser rechazada por su profesora, en quien cree a ciegas y a quien admira, quien cumple el rol en aquella época de ser una de las figuras más importantes en la vida de cada niño/a, y a quien se reconoce desde su posición privilegiada de dueña de los saberes por los que se asiste al colegio? Esta profesora era el prototipo de una persona racista y clasista, mantenedora de un sistema de castas, jerárquico por naturaleza, en una época social y política que propiciaba con violencia esta forma de estratificación social. En zona fronteriza, en donde además no parecerse a lo nacional blanco, europeo, de la elite santiaguina, tener la piel morena y un origen multicultural, era sin duda una desventaja ante un grupo minoritario de compañeras rubias, de ojos claros y de familias que se vinculaban y comportaban con nuestras profesoras desde una posición privilegiada (36).

"Fuera, retírate de la sala". Decía en tono molesto la miss de inglés. "Molestas mucho, y además te cuesta aprender....". Al tiempo y como he contado anteriormente, Karen se eximió de inglés porque "tenía dificultades para aprender el idioma", y al año siguiente, se cambió a un liceo público (27). En aquella época esto no estaba sancionado. Hoy tampoco lo está suficientemente. La violencia con que la profesora de inglés situaba a Karen en una categoría de desventaja, "tú no puedes aprender inglés", porque ¿no eres blanca?, "te cuesta aprender inglés", ¿se tratará de un problema genético de la cultura aymara?, "retírate de la sala", a ti, ino te corresponde estar aquí!... esta sala es para gente blanca, y tú no lo eres, y además interrumpes la clase. ¿Son estas las ideas que en el fondo pensaba esta profesora?.

Se aprende vicariamente sin necesidad de experimentar en primera persona lo que le ocurre a otros. En aquella época, por mi edad, creo que lo que le ocurría a Karen, y que luego fue abordado por su familia en silencio, fue para mí una situación que de alguna manera aprendí a resistir con éxito. A los $7-8$ años, ¿tenemos la capacidad para diferenciarnos de lo que le está ocurriendo a nuestras mejores amigas?, O ¿'somos capaces de sentir en este caso su angustia, dolor, pesar por lo que le estaba pasando? Era más fácil evadir la situación, no hablarlo, pero eso no significaba que no lo estuviéramos viviendo en nuestra sala de clases, hacia nuestra querida amiga Karen, hacia nosotras mismas.

Han pasado los años y cuando pedí autorización a Karen para publicar esta historia en 2016, entre risas me dijo: "claro, amiga, cuenta con mi apoyo para tu trabajo. Afortunadamente, la "miss" cambió. Te cuento que un día la encontré en la calle principal de la ciudad, y me saludó por mi nombre. Al tiempo, cuando nació mi primera hija, fue a verme al hospital y me llevó un regalo. Yo creo que en aquella época yo era muy risueña y morochita. Menos mal que mis niñas salieron blanquitas..."
Momento 2. Año 2016, segundo semestre.

Esta es una nueva jornada de asistencia a uno de los ocho establecimientos en los que estamos desarrollando el proyecto Fondecyt № 1160869 .

Hoy me encuentro sentada en el último banco de la sala de clases en una escuela en zona rural en la que puedo constatar, en distintas acciones y medidas, la preocupación de los profesores y las autoridades por integrar a los niños hijos de extranjeros al establecimiento, mediante la promoción de acciones que ellos consideran que favorecen su adaptación al sistema escolar.

En este curso hay más de 40 niños que asisten diariamente a la escuela; los niños tienen entre 10 y 11 años. La sala, el entorno, la claridad que entra por sus ventanas desde ambos costados de la sala, la actitud de los niños, silenciosa, alegre, tranquila, hace que el estar allí realizando la observación de sus interacciones sea definitivamente un agrado, en donde aparentemente todo transcurre en perfecta calma y armonía. Asisto en reiteradas ocasiones a distintas clases de este curso. Los niños han sido los últimos que han firmado documentos de asentimiento informado en donde les hemos explicado nuestra presencia en la sala de clases. De todos modos ellos se acercan a mí para preguntarme qué estoy haciendo y qué escribo en mi libreta. Ellos me ven tomar fotografías, escribir, conversar con ellos en el recreo, dejarles la cámara para que fotografíen sus lugares favoritos en el patio durante los recreos.

Según me cuenta la profesora a cargo del curso, los niños son en su mayoría hijos de peruanos y bolivianos que trabajan en la agricultura de uno de los valles de la ciudad. Los niños siempre vienen - dice la profesora- casi no registran inasistencias.

Los niños están en una clase con uno de los profesores e ininterrumpidamente transitan entre un módulo y otro. Dos niños sentados cerca de mi banco se ven muy inquietos y hacen ruidos al mover sus sillas y mesas. Veo que el profesor les llama la atención, mientras ellos no dejan de darse patadas por debajo de la mesa, mirando de reojo al profesor para no ser descubiertos. Pero al pegarse, los bancos se mueven y chocan entre sí, hacen ruido, el profesor les descubre. Les llama la atención una y otra vez. Los niños siguen con los pies por debajo. Se ven enojados, ninguno quiere rendirse, entonces los golpes aumentan dentro de lo posible en intensidad y rapidez. El profesor nuevamente les Ilama la atención. Otra vez, y otra. El profesor se ve interrumpido por estos niños que no dejan de pegarse. $\mathrm{El}$ profesor no puede seguir con su clase. Los niños siguen peleando, ¿quién quiere ser el que se dé por vencido en esta disputa? Difícil respuesta, los niños no quieren perder.

El profesor se ve serio y molesto, y eleva el tono de su voz mientras sigue enseñando al resto del curso que atiende tranquilamente, como si la escena de los dos niños en disputa no estuviese ocurriendo en ese mismo escenario. Los niños no le hacen caso y la clase no puede transcurrir en la tranquilidad que caracteriza la vida cotidiana de esta escuela. Entonces el profesor decide pedirles que se retiren de la sala: Juan, y Pedro, isalgan de la sala ahora!. Los niños se quedan quietos sin mo- 
verse. EL profesor insiste: Juan y Pedro, se van ahora. Juan es un niño de piel morena sin rasgos indígenas aparentes y posee apellidos chilenos no aymaras, a diferencia de Pedro que tiene rasgos andinos y los dos apellidos Aymara. Los niños se paran muy serios, caminan con una actitud de desgano, y se dirigen a la puerta. Al aproximarse a la puerta " Juan", dice el profesor, "te quedas en el patio, y tú, Pedro, ite vas a la sala de al lado a trabajar en integración ${ }^{2 ”}$.

Pedro mira al suelo y frunce el ceño. Juan sale mirando a sus compañeros, pero Pedro mira el suelo.

Termina la clase, se inicia el recreo. Al inicio de la clase siguiente Juan y Pedro se incorporan con sus compañeros. Regresamos a la sala de clases, y Juan entra más tranquilo, al igual que Pedro. Pedro me mira de reojo y nuevamente mira al suelo. Parece avergonzado.

Pienso: “Ustedes tienen que ubicarse, están en clases, deben obedecer al profesor, y a tí, que además te cuesta aprender, el castigo social que recibirás para extinguir tu conducta, es pedirte que vayas a nivelación". Esto, frente de tus compañeros de curso que observan como espectadores en primera persona esta escena esperando continuar con la clase. Esta escena me remite a Karen cuando la profesora le decía "tú tienes problemas para aprender". ¿No es esta la peor sanción de todas las que se le puede transmitir a los niños que presentan dificultades para aprender y que son excluidos de la sala de clases para ir a abordar los problemas de aprendizaje que tienen?.

Pero al mismo tiempo pienso en el caso del profesor: estoy preocupado por este estudiante, porque no logro que se concentre, pierde el tiempo en clases, aprovecho de pedirle que vaya a trabajar con la profesora de apoyo en integración para que así no pierda el tiempo de esta clase.

Estoy allí, no puedo intervenir. ¿Cómo se me ocurriría hacerlo allí? Sí tengo una autorización para observar las interacciones en la sala de clase. Por otro lado, es muy fácil conectar en este momento con mi yo de estudiante de enseñanza básica, y sentir angustia por lo que estoy viendo-viviendo. Pienso en el futuro de Pedro, y en cómo será su desempeño en la escuela dependiendo de la lectura que él haga a situaciones como la que está viviendo y por la que no se debe sentir orgulloso. Veo a Pedro en el futuro, dedicado a la agricultura como opción, o como estudiante de alguna de las carreras que imparte la universidad en la que trabajo. Las dos opciones serán buenas si son realmente sus opciones en un sistema educacional que determina las vidas de sus estudiantes, esto en un país que no cuenta con políticas públicas que aborden la integración intercultural de los niños chilenos con y sin ascendencia andina o afro y los niños hijos de inmigrantes de distintas nacionalidades.

En las escuelas las situaciones complejas de entender parecen

2 Programa gubernamental para niños que presentan dificultades de aprendizaje tener dos o más formas de ser leídas. Como sea, desde la observación realizada no puedo saber cuál de estos dos mensajes fue el que leyó el estudiante de ascendencia aymara expulsado de la sala de clases. Tampoco cuál es la intención de este profesor que tras una serie de observaciones, demuestra un alto compromiso con el curso y estrategias pedagógicas efectivas.

Pienso en el proceso detrás del abordaje de la interculturalidad como un problema que debería ser abordado de manera sistemática, y cómo a partir de nuestras propias biografías podemos desarrollar un enfoque desde la humanización de los conceptos, haciendo sentido a nuestras experiencias en relación a las experiencias de las personas que estudiamos, partiendo por una revisión de nuestras propias biografías de vida. ¿Qué tan conscientes o inconscientes somos sobre nuestros actos, percepciones y acciones que realizamos en relación a un otro según su origen evidenciado en la piel, los rasgos, la nacionalidad y los códigos culturales que manejamos como propios? Pienso en el impacto que acciones como las que transcurren en el teatro de la sala de clases pueden tener en la vida de una persona. Pienso en cómo la investigación social en las escuelas debe ser una herramienta de transformación social para promover el respeto, la equidad y la integración entre los niños y las niñas sin distinción de "raza", clase, género, nacionalidad. Al mismo tiempo, qué tan colonizada es nuestra forma de observar cuando investigamos a otro, ahora nacional, pero que seguimos viendo como inmigrante con todo lo que se ha evidenciado es atribuído al concepto (37).

\section{REFLEXIONES FINALES}

Experiencias de investigación que evidencian discriminación, estigmatización, y racismo, pero también integración, apoyo mutuo, asimilación, valoración de un otro hijo de inmigrante, se relevan en este proyecto y con este tipo de metodologías para producir conocimientos y romper silencios sobre situaciones que podrán ser o no replicables pero que tratan de momentos significativos en la vida de las personas que son estudiadas.

Escribir texto performativo en base a la metodología aquí propuesta, y para el caso de las vidas de niños de distintas culturas, trayectorias, formas de ver la vida y de comunicarse, y que transcurren en escuelas de la ciudad fronteriza de Arica, es una herramienta que puede ser muy útil para conectar las teorías a la base del proyecto con todo el conocimiento local, a partir de un enfoque integrador, humano, y contextual. A través de las historias que se pueden contar, nos movemos desde el cuerpo que encarna las experiencias en una línea de pedagogía crítica que llega al papel buscando performar las vidas de las personas que participan como actores, y también hacia las vidas de las audiencias que leen estos textos. Estos textos, que como Denzin sostiene, incluyen personas representadas como historias personales, escenas, lugares o el contexto en el que la historia ocurre, y una epifanía o crisis que provee la tensión dramática, son ahora una de las líneas de desarrollo del proyecto Fondecyt para obtener conocimientos inéditos de la vida cotidiana escolar y la conformación del habitus en las escuelas de la ciudad fronteriza de Arica (3). En este proyecto se trabaja en dos 
líneas, una línea que desarrolla una metodología cualitativa más bien del tipo mixta sociológica con el uso de Nvivo para la operativización de las dimensiones sociológicas que son estudiadas, y al mismo tiempo, esta línea reflexiva. Ambas líneas de desarrollo metodológico serán ensambladas para obtener ese cuadro nítido, complejo, profundo en sus matices, abstracto, con partes inconclusas pero definidas, con capacidad de evocar, transportar, conectar, icónico, simbólico, dibujado desde las voces de los niños y los actores claves que participan de la teatralidad que se desarrolla cotidianamente en las escuelas, y desde mi voz como investigadora nacida y criada en la región fronteriza de Arica, que cruza fronteras, observa y asimila desde y en distintas culturas. Desde ambos caminos se está trabajando en la representación del fenómeno que estudiamos con suficiente profundidad y rigurosidad metodológica para entender cómo se desarrollan las vidas de los niños hijos de inmigrantes con los niños chilenos en la ciudad fronteriza de Arica en la conformación de sus habitus, que hasta el momento, se ha observado son bastante diferentes a otros contextos internacionales, nacionales e incluso locales.

\section{AGRADECIMIENTOS}

Se agradece el apoyo recibido de la Universidad de Tarapacá a través del Convenio de desempeño UTA-Mineduc en su línea de Espacios Relacionales y Calidad de Vida. Este apoyo hizo posible la realización de la Escuela de verano en metodologías cualitativas para la transformación social en zona fronteriza, desde donde surge este trabajo.

Cómo citar este artículo: Zapata Sepúlveda, P. La autoetnografía interpretativa para humanizar la investigación social en contextos transfronterizos latinoamericanos. Enfermeria (Montev.). [Internet]. 2017 Oct [citado xxxxx]; 6 49-58. Disponible en: https://doi.org/10.22235/ech. v6iEspecial. 1451

\section{REFERENCIAS BIBLIOGRÁFICAS}

1. Denzin NK, Lincoln YS (Eds.). The SAGE handbook of qualitative research. 4th ed. Thousand Oaks: Sage; 2011.

2. Denzin NK. The Death of Data in Neo-Liberal Times. Paper presented at the International Congress of Qualitative Inquiry, Urbana-Champaign University of Illinois; May, 2016.

3. Denzin NK. Interpretive autoethnography (2nd ed.). Los Angeles: Sage; 2014.

4. Richardson L, St. Pierre E. Writing as a method of inquiry. In N. Denzin and Y. Lincoln (Eds.), The Sage Handbook of Qualitative Research. Thousand Oaks: Sage; 2005.

5. Reichardt CHS, Cook TD. Qualitative and quantitative methods in evaluation research. Beverly Hills: Sage; 1982.

6. Giroux HA. Beyond Dystopian Education in a Neoliberal Society. Fast Capitalism, 2013;10(1). Disponible en https:// www.uta.edu/huma/agger/fastcapitalism/10_1/home. html
7. Spinak E. Quantitative Analyses of Scientific Literature and their validity for judging Latin American production. Bull. of PAHO. 1996; 29(4): 352-360.

8. Ellis C. The ethnographic I: A methodological novel about teaching and doing autoethnography. Walnut Creek: AltaMira; 2004.

9. Richardson L. Writing: A method of inquiry. En NK Denzin, YS Lincoln (Eds.), Collecting and interpreting qualitative materials. Thousand Oaks: Sage; 1998. p. 345-371).

10. Richardson L. Getting personal: Writing stories. International Journal of Qualitative Studies in Education, 2001;14(1): 33-38.

11. Denzin NK. Interpretive biography. Newbury Park: Sage; 1989.

12. Conquergood D. Performance Studies Interventions and Radical Research. The Drama Review 2002;46:145-156. Disponible en http://www.csun.edu/ vcspc00g/301/psinterventions-tdr.pdf

13. Cayo G, Pérez L, Zapata L. Rueda de valoración familiar: un aporte constructivista a la intervención en familia. Límite: Revista de filosofía y psicología 2000(7): 27-37.

14. Zapata P. Efectos emocionales, afectivos y sociales de la privación de libertad y tortura por motivos políticos en Chile de 1973 a 1990 [Tesis doctoral]. Universidad de Salamanca, España; 2008.

15. Zapata-Sepúlveda P. Breaking my academic silence to start again making sense of why I am here, Moving from the thin to thick. Thinking about trauma and loss. Qualitative Inquiry, 2012; 18: 643-650.

16. Denzin NK. Programa. 13th International Congress of Qualitative Inquiry. University of Illinois at Urbana-Champaign. (actualizado 2017 abril 17) Disponible en http:// icqi.org/wp-content/uploads/2013/02/QI2017-Final-Program-04172017.pdf

17. Aguirre-Armendáriz E. Cuando contar la tesis es hacer la tesis. Investigación y escritura autoetnográfica. México: Centro Latinoamericano de Pensamiento Crítico; 2015.

18. Benard S. Atrapada el provincia. Un ejercicio autoetnográfico de imaginación sociológica. México: Universidad Autónoma de Aguascalientes; 2014.

19. Diversi M., Moreira C. Betweener talk. Decolonizing knowledge production, pedagogy \& praxis. Walnut Creek: Left Coast Press; 2009.

20. Pelias R. Performance: An Alphabet of Performance Writing. Walnut Creek: LeftCoast Press; 2014.

21. Brown RN, Carducci R, Kurby CR. Introduction. En RN Brown, R Carducci, CR Kurby (Eds.). Disrupting qualitative Inquiry: Possibilities and tensions in educational research. New York: Peter Lang; 2014. p. 1-31.

22. Pe-Pua R. Sikolohiyang Pilipino (Filipino psychology): A legacy of Virgilio G. Enriquez. Asian Journal of Social Psychology 2000; 3:49-71.

23. Denzin NK, Lincoln YS, Smith LT (Eds.). Handbook of Critical and Indigenous Methodologies. Thousand Oaks, CA: Sage; 2008.

24. Tamas S. Life After Leaving: The Remains of Spousal Abuse (Writing Lives: Ethnographic Narratives). Walnut Creek: Left Coast Press Inc.; 2011. 
25. Nguyen NHC. Memory is another country: Women of the Vietnamese diaspora. Santa Barbara: Praeger; 2009.

26. Zapata-Sepúlveda $P$, López-Sánchez F, Sánchez-Gómez M. Concepción de mundo, aspectos contextuales y bienestar psicológico en chilenos supervivientes a experiencias de prisión y tortura por motivos políticos a más de 30 años de ocurridos los hechos. Universitas Psychologica 2009; 8(3):761-769.

27. Zapata-Sepúlveda P. The Power of Saying the Normally "Unsaid" as an Act of Empowering a Woman's Voice in the Academia and the Fictional Parallel Side Behind This Power in a Global Era. Qualitative Inquiry, first online 2016. Disponible en http://journals.sagepub.com/doi/ full/10.1177/1077800416659087

28. Zapata-Sepúlveda P. Experiencias de investigación, desafíos y limitaciones en el trabajo con inmigrantes y refugiados en la ciudad fronteriza de Arica. En: ME Tijoux (Ed.) Racismo en Chile: la piel como marca de la inmigración. Santiago: Editorial Universitaria; 2016. p. 141-158.

29. Zapata-Sepúlveda P. The overcoming of the attacks on freedom of speech through qualitative research. International Journal of Qualitative Research (en prensa).

30. Muñoz KL. Prefacio. En: KL Muñoz. Transcribing Silence. Culture, relationships, and communitation. Walnut Creek: Left Coast Press Inc. ; 2014: 7-14.
31. Zapata-Sepúlveda P, Tijoux ME, Espinoza-Lobos M. La investigación critica como una forma de romper el cristal entre las prácticas "comunes" en la academia pública chilena y el caso de los estudiantes migrantes latinoamericanos de color en nuestras salas de clases: un llamado a humanizar, un llamado a reflexionar. Diálogo andino 2015(47): 71-81. Disponible en https://dx.doi.org/10.4067/S071926812015000200008

32. Pelias R. Writing performance: Poeticizing the researcher's body. Carbondale: Southern Illinois University Press; 1999.

33. Pollock D. Performing writing. En: P Phelan J Lane (Eds.). The ends of performance. New York: New York University Press; 1998: 73-193.

34. Moreira C. I hate chicken breast: a tale of resisting stories and disembodied knowledge construction. International Journal of Qualitative Studies en Education 2012, 25(2): 151-167.

35. Bidou J, Guzmán P. Salvador Allende. [Video] Belgium: JBA Productions; 2004.

36. González S. El Dios Cautivo. Las Ligas Patrióticas en la chilenización compulsiva de Tarapacá (1910-1922). Santiago de Chile: LOM; 2004.

37. Tijoux M. (Ed.). Racismo en Chile: La piel como marca de la inmigración. Santiago de Chile: LOM; 2016. 